Texas Southern University

Digital Scholarship @ Texas Southern University

Faculty Publications

$1-1-2019$

\title{
Symmetry of generalized rivalry network models determines patterns of interocular grouping in four-location binocular rivalry
}

\author{
Martin Golubitsky \\ The Ohio State University \\ Yukai Zhao \\ The Ohio State University \\ Yunjiao Wang \\ Texas Southern University \\ Zhong Lin Lu \\ NYU Shanghai
}

Follow this and additional works at: https://digitalscholarship.tsu.edu/facpubs

\section{Recommended Citation}

Golubitsky, Martin; Zhao, Yukai; Wang, Yunjiao; and Lu, Zhong Lin, "Symmetry of generalized rivalry network models determines patterns of interocular grouping in four-location binocular rivalry" (2019). Faculty Publications. 130.

https://digitalscholarship.tsu.edu/facpubs/130

This Article is brought to you for free and open access by Digital Scholarship @ Texas Southern University. It has been accepted for inclusion in Faculty Publications by an authorized administrator of Digital Scholarship @ Texas Southern University. For more information, please contact haiying.li@tsu.edu. 


\title{
RESEARCH ARTICLE | 50 Years of Modeling Neural Activity: Celebrating Jack Cowan's Career
}

\section{Symmetry of generalized rivalry network models determines patterns of interocular grouping in four-location binocular rivalry}

\author{
Martin Golubitsky, ${ }^{1}$ (ㄷ) Yukai Zhao, ${ }^{2}$ Yunjiao Wang, ${ }^{3}$ and Zhong-Lin $\mathrm{Lu}^{\mathbf{4}, \mathbf{5 , 6}}$ \\ ${ }^{1}$ Department of Mathematics, The Ohio State University, Columbus, Ohio; ${ }^{2}$ Department of Psychology, The Ohio State \\ University, Columbus, Ohio; ${ }^{3}$ Department of Mathematics, Texas Southern University, Houston, Texas; ${ }^{4}$ Division of Arts and \\ Sciences, NYU Shanghai, Shanghai, China; ${ }^{5}$ Center for Neural Science and Department of Psychology, New York University, \\ New York, New York; and ${ }^{6} \mathrm{NYU}$-ECNU Institute of Brain and Cognitive Neuroscience, Shanghai, China
}

Submitted 10 July 2019; accepted in final form 22 August 2019

Golubitsky M, Zhao Y, Wang Y, Lu ZL. Symmetry of generalized rivalry network models determines patterns of interocular grouping in four-location binocular rivalry. J Neurophysiol 122: 1989_ 1999, 2019. First published September 18, 2019; doi:10.1152/jn. 00438.2019.- Previously, symmetry of network models has been proposed to account for interocular grouping during binocular rivalry. Here, we construct and analyze generalized rivalry network models with different types of symmetry (based on different kinds of excitatory coupling) to derive predictions of possible perceptual states in 12 experiments with four retinal locations. Percepts in binocular rivalry involving more than three locations have not been empirically investigated due to the difficulty in reporting simultaneous percepts at multiple locations. Here, we develop a novel reporting procedure in which the stimulus disappears when the subject is cued to report the simultaneously perceived colors in all four retinal locations. This procedure ensures that simultaneous rather than sequential percepts are reported. The procedure was applied in 12 experiments with six binocular rivalry stimulus configurations, all consisting of dichoptic displays of red and green squares at four locations. We call configurations with an even or odd number of red squares even or odd configurations, respectively. In experiments using even stimulus configurations, we found that even percepts were more frequently observed than odd percepts, whereas in experiments using odd stimulus configurations even and odd percepts were observed with equal probability. The generalized rivalry network models in which couplings depend on stimulus features and spatial configurations was in better agreement with the empirical results. We conclude that the excitatory coupling strength in the horizontal and vertical configurations are different and the coupling strengths between the same color and between different colors are different.

NEW \& NOTEWORTHY Wilson network models of interocular groupings during binocular rivalry are constructed by considering features that indicate equal coupling strengths. Network symmetries, based on equal couplings, predict percepts. For a four-location rivalry experiment with red or green squares at each location, we analyze different possible Wilson networks. In our experiments we develop a novel reporting procedure and show that networks in which stimulus features and spatial configurations are distinguished best agree with experiments.

Address for reprint requests and other correspondence: M. Golubitsky, Department of Mathematics, The Ohio State University, Columbus, OH 43210 (e-mail: golubitsky.4@osu.edu). binocular rivalry; Hopf bifurcation; interocular grouping; symmetry; Wilson networks

\section{INTRODUCTION}

When corresponding retinal locations of the two eyes receive different monocular images, one often perceives alternation of the two monocular images. The phenomenon, called binocular rivalry, was first discovered by Porta in the 16th century. It has been studied extensively by vision scientists (Wade 1998) and recently has been exploited to study consciousness and visual processing outside of awareness (Blake 2001; Blake and Wilson 2011). Originally considered as a phenomenon reflecting ocular competition, later studies demonstrated that binocular rivalry could also reflect feature competition (Logothetis et al. 1996). Large rival stimuli often lead to so-called piecemeal rivalry, in which one perceives a mosaic consisting of intermingled portions of both monocular images (Blake et al. 1992; Hollins and Hudnell 1980; Meenes 1930; O'Shea et al. 1994, 1997), although complete monocular dominance does occur significantly more often than chance (Blake et al. 1992). With binocular rivalry displays that consisted of multiple targets (e.g., green and red circles) in multiple locations, a very interesting phenomenon, called "interocular grouping," was discovered: Multiple small rival targets scattered throughout the visual field engaged in synchronized alternations such that all targets of a particular feature (e.g., all the green circles), independent of the eye of origin, became dominant simultaneously (Alais and Blake 1999; Drenhaus 1975; Kovács et al. 1996; Whittle et al. 1968). These observations suggest that binocular rivalry may reflect both ocular and stimulus feature interactions (Blake and Wilson 2011).

In many binocular displays with multiple targets and retinal locations (attributes), targets with two different stimulus features are directed into each of the two eyes (e.g., one red and one green) at each retinal location. If there is only interocular competition in the visual system at each retinal location, a binocular rivalry display with $N$ locations would generate $2^{N}$ perceptual states, which is the number of all possible combi- 
nations of the percept at each retinal location. The prominence of interocular grouping seems to suggest that some of the perceptual states might be perceived more often than others. In this study, we ask: Are some of the potential perceptual states more probable than others? What can the relative frequencies of the different perceptual states tell us about neural processing?

The original binocular rivalry displays (Alais and Blake 1999; Drenhaus 1975; Kovács et al. 1996; Whittle et al. 1968) used to demonstrate interocular grouping involved many retinal locations and a large number of potential perceptual states. Although some extremely interesting observations on interocular grouping were made from those displays, it is impossible to obtain detailed measures of the perceptual states during binocular rivalry because reporting simultaneous percepts at all the retinal locations is very difficult or impossible. To reduce the number of required responses, Tong et al. (2006) suggested a simplified version of the colored disks experiments in Kovács et al. (1996) in which two monocular images each with four colored disks are shown to the subject. To our knowledge no one has conducted an experiment with such displays, perhaps because reporting simultaneous percepts at four locations is still difficult.

In this study, we developed a novel reporting procedure for four-location binocular rivalry stimuli, in which the stimulus disappeared when the subject was cued to report the simultaneously perceived colors in all four retinal locations to ensure that simultaneous rather than sequential percepts were reported. Six binocular rivalry configurations, all consisting of dichoptic displays of red and green squares at four retinal locations, were used in the study, including all the same color in each eye, equal number of red and green squares arranged in diagonal, horizontal and vertical configurations in each eye, and unequal numbers of red and green squares in each eye. Prior to the experiment, we constructed and analyzed generalized rivalry network models with different types of symmetry to derive predictions of possible perceptual states in these binocular displays. The results of the experiments allowed us to specify the symmetry in the neural architecture of binocular rivalry.

\section{GENERALIZED RIVALRY NETWORK MODELS}

A number of mathematical theories and computational models have been proposed to account for the rich phenomena in binocular rivalry (Alais 2012; Brascamp et al. 2015; Dayan 1998; Freeman 2005; Hayashi et al. 2004; Hohwy et al. 2008; Kakimoto and Aihara 2009; Kang and Blake 2011; Kovács et al. 1996; Said and Heeger 2013; Stollenwerk and Bode 2003; Wilson 2007, 2009, 2010). Although each theory or model provides unique insights on certain aspects of visual processing

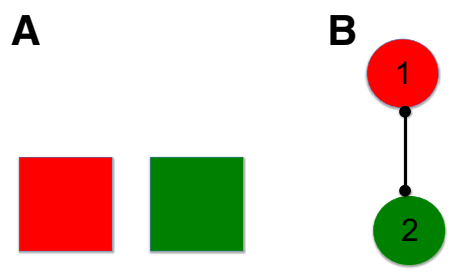

Fig. 1. A (configuration $A$ in Fig. 7): left eye and right eye images of one-location experiment with two possible colors (red and green). $B$ : Wilson network with one location column and two levels. Arrows with end stop indicate inhibitory coupling.

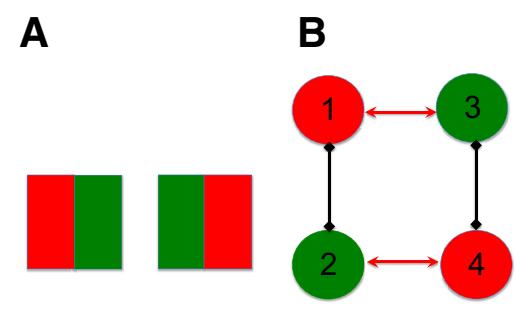

Fig. 2. A (configuration $B$ in Fig. 7) left eye and right eye images of two-location experiment with two possible colors (red and green) in each location. $B$ : Wilson network with two location columns and two levels in each column. Red solid arrows indicate excitatory coupling between different colors in two horizontally placed locations. Arrows with end stop indicate inhibitory coupling.

related to rivalry, all share many of the principles of the generalized rivalry network model proposed by Hugh Wilson (Wilson 2007, 2009, 2010). In addition, the generalized rivalry network model ("Wilson networks") has also received a tremendous amount of empirical support in accounting for the dynamics of binocular rivalry (Brascamp et al. 2015; Dieter et al. 2017; Klink et al. 2008, 2010; Richard et al. 2018; Seely and Chow 2011; van Ee 2009; Wilson 2017). Here, we construct and analyze symmetries of generalized rivalry network models in a number of four-location binocular rivalry experiments. Our intention is to derive predictions of percepts that we expect to occur more frequently in these experiments.

Generalized rivalry network models consist of nodes that represent neuronal populations and arrows that indicate coupling (excitatory or inhibitory) between the nodes, organized as a set of attribute columns, each representing one attribute of an external image. Specifically, in the models of our experiments, each column in the network represents a spatial location and consists of two nodes, each representing a feature (red or green) of the image at that spatial location. At any moment of time, according to the model, the percept experienced by an observer corresponds to the dominant activation pattern of the network.

Wilson networks have two types of coupling. First, there is inhibitory coupling between the two nodes in the same attribute column. These couplings represent stimuli from the two eyes in each retinal location; the inhibitory couplings in Figs. $1 B, 2 B$, $3 B, 4 B, 5 B$, and $6 B$ are indicated by arrows with end stops.

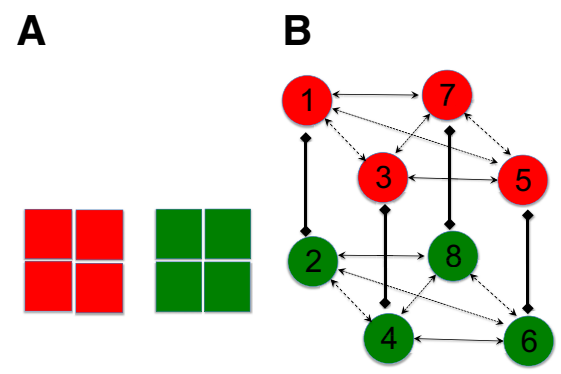

Fig. 3. A (configuration $C$ in Fig. 7): left eye and right eye images of four-location pure color even experiment with two possible levels in each location (red and green). $B$ : Wilson network with four location columns and two levels in each column. Black solid arrow indicates excitatory coupling between same color in two horizontally placed locations. Black long-dashed arrow indicates excitatory coupling between same color in two vertically placed locations. Black short-dashed arrow indicates excitatory coupling between same color in two diagonally placed locations. Symmetry group is $\Gamma=$ $\mathbf{Z}_{2}(\rho) \times \mathbf{Z}_{2}(\tau) \times \mathbf{Z}_{2}(\kappa)$, where $\rho, \tau$, and $\kappa$ are defined in $E q$. 5. Arrows with end stop indicate inhibitory coupling. 
A

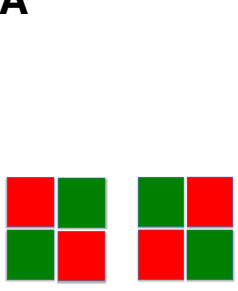

B

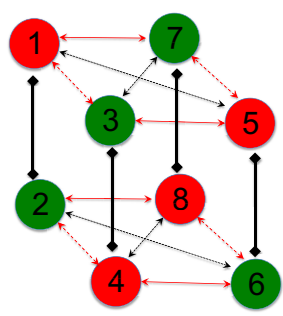

Fig. 4. A (configuration $F$ in Fig. 7): images in diagonal even colored-square experiment (Kovács et al. 1996; Tong et al. 2006). A: left eye and right eye images of four-location experiment with two possible levels in each location (red and green). B: Wilson network with four location columns and two levels in each column. Red solid arrow indicates excitatory coupling between different colors in two horizontally placed locations. Red long-dashed arrow indicates excitatory coupling between different colors in two vertically placed locations. Black short-dashed arrow indicates excitatory coupling between same color in two diagonally placed locations. Symmetry group is $\Gamma=$ $\mathbf{Z}_{2}(\rho) \times \mathbf{Z}_{2}(\tau) \times \mathbf{Z}_{2}(\kappa)$, where $\rho, \tau$, and $\kappa$ are defined in $E q$. 5. Arrows with end stop indicate inhibitory coupling.

Motivated by ocular competition in binocular rivalry, the inhibitory coupling tends to promote winner-take-all dynamics in each retinal location; that is, if the activity level in any one retinal location is maximum at time $t$, then in the absence of excitatory coupling from other columns that value will tend to continue to be the maximum in forward time. Second, through Hebbian learning, the dichoptic displays promote all-to-all excitatory coupling between nodes within each eye, corresponding to the patterns on which the network is trained. These patterns are called learned patterns. Diekman and Golubitsky (2014) observed that the strength of the different excitatory couplings in Wilson network models may well depend on image features. For example, the Hebbian learning-induced coupling between two nodes in the same eye might depend on whether the nodes being connected are stimulated by the same colors or different colors or the geometric orientation (horizontal, vertical, or diagonal) of connection (see, e.g., Fig. 4B). In particular, the symmetry group of the Wilson network can change depending on whether the various couplings are the same or different.
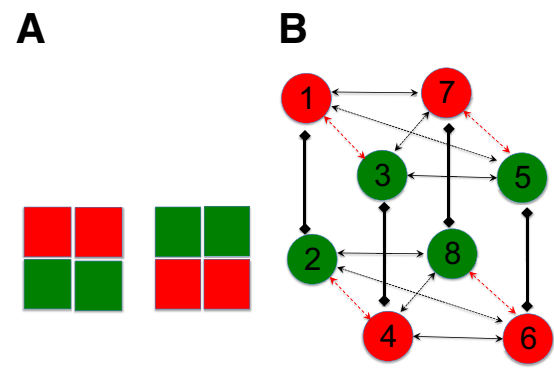

Fig. 5. A (configuration $E$ in Fig. 7): images in horizontal even colored-square experiment (Kovács et al. 1996; Tong et al. 2006). A: left eye and right eye images of four-location experiment with two possible levels in each location (red and green). $B$ : Wilson network with four location columns and two levels in each column. Black solid arrow indicates excitatory coupling between same color in two horizontally placed locations. Red long-dashed arrow indicates excitatory coupling between different colors in two vertically placed locations. Red short-dashed arrow indicates excitatory coupling between different colors in two diagonally placed locations. Symmetry group is $\Gamma=$ $\mathbf{Z}_{2}(\rho) \times \mathbf{Z}_{2}(\tau) \times \mathbf{Z}_{2}(\kappa)$, where $\rho, \tau$, and $\kappa$ are defined in $E q$. 5. Arrows with end stop indicate inhibitory coupling.

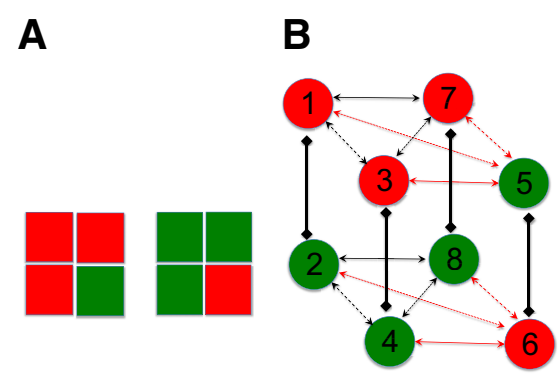

Fig. 6. A (configuration $H$ in Fig. 7): images in odd colored-square experiment proposed in Diekman and Golubitsky (2014). Left eye and right eye images of four-location experiment with two possible levels in each location (red and green). $B$ : Wilson network with four location columns and two levels in each column. Lines with arrow heads indicate excitatory coupling and lines with end stop heads indicate inhibitory coupling. Black indicates arrows that connect nodes of the same color. Red indicates arrows that connect nodes of different colors. Solid arrows indicate horizontal coupling; long-dashed arrows indicate vertical coupling; and short-dashed arrows indicate diagonal coupling. Symmetry group is $\Gamma=\mathbf{Z}_{2}(\rho)$, where $\rho$ is defined in $E q$. 5 .

In general, it is difficult to solve the differential equations of the generalized rivalry network models analytically and Wilson proceeded using simulations of Wilson-Cowan equations (Wilson 2007, 2009, 2010). As discussed in Diekman et al. $(2012,2013)$ and Diekman and Golubitsky (2014), progress on understanding possible solution types, that is, possible perceptual states from such network models, can be made by analyzing the symmetries and the symmetry-breaking bifurcations in such networks. The basic idea is to enumerate the kinds of rigid phase-shift synchrony that small-amplitude periodic solutions can have in the class of network equations. This list — or menu - gives a prediction of possible perceptual states. Because rigid phase-shift synchrony is a product of network symmetry (Golubitsky et al. 2012; Stewart and Parker 2007) and network symmetries are based on node types and types of inhibitory and excitatory coupling between nodes, we can predict possible perceptual states from the generalized rivalry networks and compare them with empirical observations. Our hypothesis is that the percepts that are specified by symmetry breaking will appear more frequently in the experiments than will the percepts that are not. This kind of assumption is discussed in Golubitsky and Stewart (2002).

In this section, we follow (Diekman et al. 2012, 2013; Diekman and Golubitsky 2014) and use symmetry to predict possible perceptual states from the generalized rivalry network models. In each case, we predict a menu of possible perceptual states by classifying the symmetry types of small-amplitude periodic solutions that can be associated with alternation and can be obtained through Hopf bifurcation (see APPENDIX for an introduction). To familiarize the readers with our methodology, we first present symmetry analyses of Wilson networks in one-location and two-location rivalry experiments, then proceed to make predictions for four-location binocular rivalry experiments in six different configurations (see Fig. 7, $A-H$ ). In the first set of analysis, we assume that all types of couplings are different. We then consider reduced cases in which some couplings are the same.

\section{One-Location Binocular Rivalry}

The simplest binocular rivalry stimulus consists of dichoptic presentation of two incompatible stimulus features 


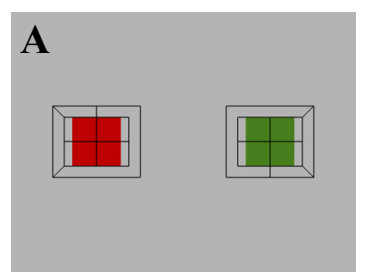

Pure Color

no-spacing (RRRR)

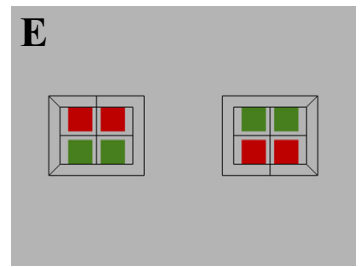

Horizontal (RGGR)

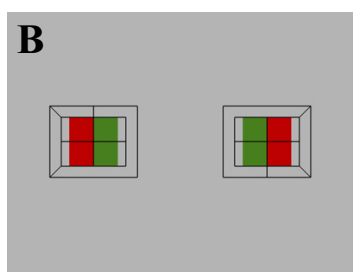

Vertical

no-spacing (RRGG)

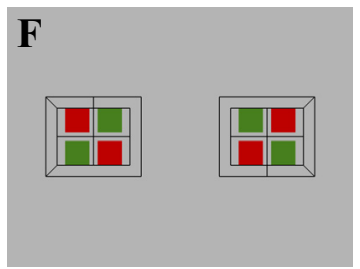

Diagonal (RGRG)

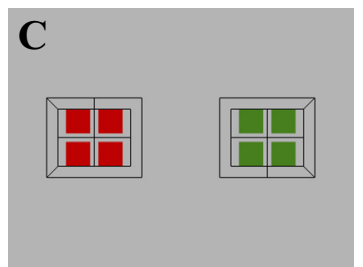

Pure Color (RRRR)

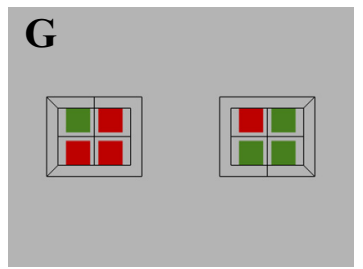

Upper Left (GRRR)

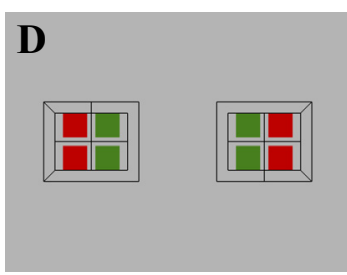

Vertical (RRGG)

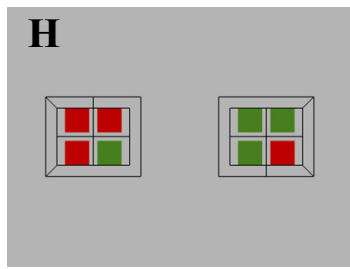

Lower Right(RRGR)

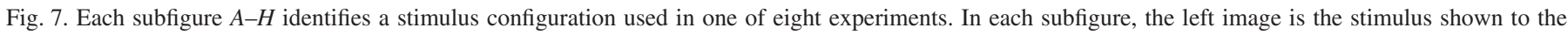

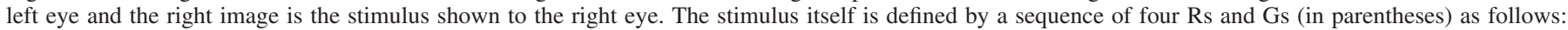
Use the left eye stimulus to list the colors (Red or Green) of the small squares in the order upper left, lower left, lower right, upper right.

at one retinal location (Fig. $1 A)$. The resulting bistable perceptual rivalry can be captured by a simple Wilson network with one attribute column and two mutually inhibiting nodes.

The activity of the two nodes is often described by the following system of differential equations (Curtu et al. 2008; Diekman et al. 2012; Laing and Chow 2002; Shpiro et al. 2007):

$$
\begin{aligned}
& \varepsilon \dot{E}_{1}=-E_{1}+G\left(I_{1}-\beta E_{2}-g H_{1}\right) \\
& \dot{H}_{1}=E_{1}-H_{1} \\
& \varepsilon \dot{E}_{2}=-E_{2}+G\left(I_{2}-\beta E_{1}-g H_{2}\right) \\
& \dot{H}_{2}=E_{2}-H_{2}
\end{aligned}
$$

where the activity of each node is described by a firing rate variable $E_{i}$ and a rate adaptation variable $H_{i}$ for $i=1,2$. $G$ is a sigmoid function that models the effect of all inputs to the node; $g$ is the strength of adaptation and $\varepsilon$ is the ratio of time scales on which $E$ and $H$ evolve. The mutual inhibition due to conflict information in the two eyes is described by $\beta$ and input strength, modeled by $I_{i}$ for $i=1,2$. Note that when $I_{1}=I_{2}$, the system has $\mathbf{Z}_{2}$ symmetry. The case with $I_{1} \neq I_{2}$ can be viewed as a system resulting from perturbation to the symmetric system with $I_{1}=I_{2}$. Since the symmetry can simplify our analysis, we assume $I_{1}=I=I_{2}$.

In this model, the stimulus in the right eye is perceived when $E_{2}>E_{1}$; the stimulus in the left eye is perceived when $E_{2}<$ $E_{1}$. Equality in activity levels is called fusion. Moreover, a solution to Eq. 1 corresponds to perceptual alternation when the direction of the inequality changes in time. As noted in Diekman et al. (2012), perceptual alternation and fusion can occur robustly in periodic solutions with spatiotemporal symmetry.

We can simplify the notation by letting $X_{1}=\left(E_{1}, H_{1}\right)$ be the upper node coordinates and $X_{2}=\left(E_{2}, H_{2}\right)$ be the lower node coordinates. Then $E q .1$ has the form

$$
\begin{aligned}
& \dot{X}_{1}=F\left(X_{1}, X_{2}, I\right) \\
& \dot{X}_{2}=F\left(X_{2}, X_{1}, I\right)
\end{aligned}
$$

A network symmetry is a permutation of nodes that takes solutions to solutions. The system Eq. 2 has a permutation symmetry $\rho$ given by swapping nodes 1 and 2 ; that is,

$$
\rho\left(X_{1}, X_{2}\right)=\left(X_{2}, X_{1}\right)
$$

In shorthand, the symmetry is just the permutation $\rho$ (1 2).

This symmetry leads in a mathematically naturally way to perceptual alternation, as follows. Suppose that $X(t)$ is a $T$-periodic solution to $E q .2$ and suppose that $\sigma X(0)$ lies on the solution trajectory $\{X(t)\}$. Then uniqueness of solutions to initial value problems implies that there is a phase-shift $\theta$ between 0 and 1 such that

$$
\rho X(t)=X(t+\theta T)
$$

for all $t$. Applying $\rho$ twice yields

$$
X(t+2 \theta T)=X(t)
$$

for all $t$. Hence either $\theta=0$ or $\theta=\frac{1}{2}$.

In the first case $X_{2}(t)=X_{1}(t)$ for all $t$ and the state is a fusion state, whereas in the second case $X_{2}(t)=X_{1}\left(t+\frac{T}{2}\right)$ for all $t$. Perceptual alternation follows from the phase-shift synchrony because $E_{2}(t)>E_{1}(t)$ implies $E_{1}\left(t+\frac{T}{2}\right)>E_{2}\left(t+\frac{T}{2}\right)$. In other words, the symmetry of the network in this case predicts alternation between two possible rivalry states: the stimulus perceived in the left eye (stimulus "red square") or in the right eye (stimulus "green square").

It is an important fact that phase-shifts that are associated with network symmetries are rigid; that is, small changes in parameters in the model will perturb the periodic solutions but will not change the phase shifts (see Golubitsky and Stewart 2002). Moreover, in 
networks, rigidity of phase-shift synchrony in periodic solutions can only be caused by network symmetry (Golubitsky and Stewart 2006; Golubitsky et al. 2012).

As is true of any experiment, symmetry can only be approximate and breaking of symmetry or the inclusion of small stochastic effects can change results slightly. In this way, symmetry can lead to experimentally verifiable results and can help to explain certain aspects of experiments.

\section{Two-Location Binocular Rivalry}

Binocular rivalry with two stimulus features may involve multiple retinal locations. The monkey-jungle experiments of Kovács et al. (1996) give an example of a two-location experiment as do the experiments in Jacot-Guillarmod et al. (2017). A general two-location Wilson network analysis of this experiment was given in Diekman et al. (2013) and Diekman and Golubitsky (2014). We review those results here expressed in terms analogous to our four-location colored-square experiment.

Figure 2 shows the stimulus and Wilson network model for a binocular rivalry configuration that involves dichoptic presentation of stimuli at two retinal locations. The Wilson rivalry network model has four nodes and two attribute columns that represent neuronal populations receiving inputs from the two locations in each of the two eyes. To simplify the presentation of excitatory couplings in the Wilson network, we organize the two learned patterns by one on the top nodes and one on the bottom nodes of the attribute columns.

The activity of one of the four nodes is described by the following system of differential equations:

$$
\begin{aligned}
& \dot{H}_{1}=E_{1}-H_{1} \\
& \varepsilon \dot{E}_{1}=-E_{1}+G\left(I+\gamma_{13} E_{3}-\beta_{12} E_{2}-g_{1} H_{1}\right)
\end{aligned}
$$

where $\gamma_{13}>0$ represents the strength of the excitatory coupling (due to Hebbian learning) that associates different locations in the same learned pattern. The differential equations for the other three nodes are obtained by permuting the variables in Eq. 3 in obvious ways. We can write the equations in a form analogous to Eq. 2 as follows.

$$
\begin{aligned}
& \dot{X}_{1}=F\left(X_{1}, X_{2}, X_{3}, I\right) \\
& \dot{X}_{2}=F\left(X_{2}, X_{1}, X_{4}, I\right) \\
& \dot{X}_{3}=F\left(X_{3}, X_{4}, X_{1}, I\right) \\
& \dot{X}_{4}=F\left(X_{4}, X_{3}, X_{2}, I\right)
\end{aligned}
$$

where $X_{j}=\left(E_{j}, H_{j}\right)$ and $F$ is the right side of $E q .3$.

There are four perceptual states associated with the fournode network in Fig. $2 B$ : stimulus $\mathrm{R}$ or $\mathrm{G}$ at the left location and stimulus $R$ or $G$ at the right location. We name these percepts RR, RG, GR, GG. States in Eq. 4 predict these percepts as follows:

$$
\begin{aligned}
& \text { RG } E_{1}>E_{2} \quad E_{3}>E_{4} \\
& \text { RR } E_{1}>E_{2} \quad E_{4}>E_{3} \\
& \text { GG } E_{2}>E_{1} \quad E_{3}>E_{4} \\
& \text { GR } E_{2}>E_{1} \quad E_{4}>E_{3}
\end{aligned}
$$

This two-column network has two independent symmetries. The generalization of the one-column network symmetry that permutes the stimuli is $\rho=(12)(34)$. The new symmetry that permutes the two locations is $\tau=(12)(34)$.

With these symmetries, equivariant Hopf bifurcation generates four types of periodic solutions: two bifurcations lead to fusion states that we eliminate and two bifurcations lead to perceptually alternating states (see the APPENDIX or Diekman et al. 2013; Diekman and Golubitsky 2014; Golubitsky and Stewart 2002). As above, let $X(t)=\left(X_{1}, X_{2}, X_{3}, X_{4}\right)$ be $T$-periodic. If $\rho X(t)=X(t)$, then $X(t)$ consists of fusion states since $E_{1}(t)=$ $E_{2}(t)$ and $E_{3}(t)=E_{4}(t)$. On the other hand, if $\rho X(t)=$ $X\left(t+\frac{T}{2}\right)$, then perceptual alternation occurs. Specifically, if also $\tau X(t)=X(t)$, then the periodic solution alternates between percepts $R G$ and $G R$, whereas if $\tau X(t)=X\left(t+\frac{T}{2}\right)$, then the periodic solution alternates between percepts $R R$ and $G G$. In particular, the Wilson rivalry model predicts that four perceptual states, $R R, G G, R G, G R$, can be expected to be observed in two-location rivalry experiments with equal likelihood.

\section{Four-Location Binocular Rivalry}

The simplified version of the colored-square experiments suggested by Tong et al. (2006) consists of four retinal locations. Here, following (Diekman and Golubitsky 2014), we consider six stimulus configurations, all involving dichoptic displays consisting of squares that are either red or green with the colors interchanged between the two images. We describe them using the left eye image:

1) Pure color: Configuration has four red squares. See Fig. $7 A$ or Fig. $7 C$.

2) Diagonal: Configuration has two red squares and two green squares on diagonal lines. See Fig. $7 F$.

3) Horizontal: Configuration has two red squares and two green squares on horizontal lines. See Fig. $7 E$.

4) Vertical: Configuration has two red squares and two green squares on vertical lines. See Fig. $7 B$ or Fig. $7 D$.

5) Upper left: Configuration has one green square in upper left location and three red squares. See Fig. $7 G$.

6) Lower right: Configuration has one green square in lower right location and three red squares. See Fig. $7 H$.

The Wilson networks for these configurations have all-to-all monocular excitatory coupling between nodes in each learned pattern. Specifically, in the Wilson network figures, there are six types of excitatory coupling: horizontal (solid), vertical (long-dashed), and diagonal (short-dashed) each of which can be black (connecting two nodes stimulated by the same colors) or red (connecting two nodes stimulated by different colors).

As we will see the network symmetries of the Wilson networks are identical for the first four configurations. The interpretation of the symmetric solutions is slightly different. However, the important point is that nonfusion periodic solutions obtained by Hopf bifurcation are identical for these four configurations and have a simple description. All of the predicted perceptual states in these configurations have an even number of red squares and an even number of green squares. We call these the even percepts. In the last two configurations, the symmetries are minimal and no specific perceptual pattern is predicted. These models suggest that even percepts should appear more frequently than odd percepts in experiments using the first four stimulus configurations and even percepts and odd 
Table 1. Symmetry type of percept

\begin{tabular}{llll}
\hline \hline & \multicolumn{1}{c}{$\tau$} & \multicolumn{1}{c}{$\kappa$} & \multicolumn{1}{c}{$\rho$} \\
\hline$\alpha$ & trivial & trivial & nontrivial \\
$\beta$ & trivial & nontrivial & nontrivial \\
$\gamma$ & nontrivial & trivial & nontrivial \\
$\delta$ & nontrivial & nontrivial & nontrivial \\
\hline
\end{tabular}

percepts should be equally likely in the last two stimulus configurations.

Configuration $C$. In this configuration, one learned pattern has four red squares and the other has four green squares, as shown in Fig. $3 A$.

We model the experiment with this stimulus configuration by a Wilson network consisting of four attribute columns, where each attribute refers to the position of one of the squares (upper left, lower left, lower right, upper right) and has two levels (red, green). The eight-node Wilson network with two pure color learned patterns is shown in Fig. $3 B$.

The permutation symmetries of the Wilson network are generated by

$$
\begin{aligned}
& \rho=(12)(34)(56)(78) \text { top-bottom } \\
& \tau=(17)(28)(35)(46) \text { left-right } \\
& \kappa=(13)(24)(57)(68) \text { up-down }
\end{aligned}
$$

If $\rho$ acts trivially on a periodic state $\left[X_{1}(t), \ldots, X_{8}(t)\right]$, then

$$
X_{2}(t)=X_{1}(t) \quad X_{4}(t)=X_{3}(t) \quad X_{6}(t)=X_{5}(t) \quad X_{8}(t)=X_{7}(t)
$$

and this state is a fusion state. So, we assume $\rho$ acts nontrivially. Table 1 shows the four kinds of nonfusion periodic states given by the actions of $\tau$ and $\kappa$.

The four nonfusion state types are

$$
\begin{array}{ll}
\alpha & \left(x_{0}, x_{1 / 2} ; x_{0}, x_{1 / 2} ; x_{0}, x_{1 / 2} ; x_{0}, x_{1 / 2}\right) \\
\beta & \left(x_{0}, x_{1 / 2} ; x_{1 / 2}, x_{0} ; x_{1 / 2}, x_{0} ; x_{0}, x_{1 / 2}\right) \\
\gamma & \left(x_{0}, x_{1 / 2} ; x_{0}, x_{1 / 2} ; x_{1 / 2}, x_{0} ; x_{1 / 2}, x_{0}\right) \\
\delta & \left(x_{0}, x_{1 / 2} ; x_{1 / 2}, x_{0} ; x_{0}, x_{1 / 2} ; x_{1 / 2}, x_{0}\right)
\end{array}
$$

where $x_{0}(t)$ is $T$-periodic and $x_{1 / 2}(t)=x_{0}\left(t+\frac{T}{2}\right)$.

There are a total of eight patterns: the four patterns listed in the first column of Table 2 and the four complementary patterns obtained when $x_{0}(t)<x_{1 / 2}(t)$.

Configurations $D-F$. Each of these stimulus configurations are even states that have two red squares and two green squares. The Wilson network for the diagonal colored-square configuration is shown in Fig. 4. This network is presented so that the learned pattern couplings are in horizontal planes; that is, the red and green levels are inverted in the lower left and upper right attribute columns. Note that this network is isomorphic to the one in Fig. 3 and hence has the same symmetry group. Similarly, the four configurations (pure, diagonal, horizontal, and vertical) all have the same set of solution patterns (see Table 2) and they are characterized by having an even number of red squares and an even number of green squares.

Details of the horizontal colored-square configuration are shown in Fig. 5 with all relevant information given in the caption. The details of the vertical colored-square configura- tion are not shown; they are analogous to those of the horizontal colored-square configuration.

Configurations $G$ and $H$. The Wilson network for configuration $H$ is shown in Fig. 6. Each of these stimulus configurations has an odd number of red squares and an odd number of green squares. The only symmetry is $\mathbf{Z}_{2}(\rho)$ and Hopf bifurcation leads to solutions that alternate between a pattern and its complementary pattern. No particular pattern is preferred; all percepts are equally possible.

The even experiment states have been discussed previously; here we discuss the RGGG and GRRR rivalry experiment. The symmetry group for the associated rivalry network is just $\mathbf{Z}_{2}(\rho)$. As noted in the one-location theory, there are two types of solutions one of which is a fusion state. Thus, all nonfusion percepts are given by $\rho X(t)=X\left(t+\frac{T}{2}\right)$. Suppose

$$
X(t)=\left[x_{1}(t), x_{2}(t), x_{3}(t), x_{4}(t), x_{5}(t), x_{6}(t), x_{7}(t), x_{8}(t)\right] .
$$

Then, $\rho$ symmetry implies

$$
\begin{aligned}
& x_{2}(t)=x_{1}\left(t+\frac{T}{2}\right) \\
& x_{4}(t)=x_{3}\left(t+\frac{T}{2}\right) \\
& x_{6}(t)=x_{5}\left(t+\frac{T}{2}\right) \\
& x_{8}(t)=x_{7}\left(t+\frac{T}{2}\right)
\end{aligned}
$$

The percepts associated with these solutions have alternation in each column but at different times. Therefore, any possible percept can appear, and all percepts appear with equal likelihood.

\section{Changes in Model Features Lead to Changes in Symmetry}

Diekman and Golubitsky (2014) observe that the process of modeling rivalry by Wilson networks has two parts:

1) Determine the attributes columns and those columns that are the same.

2) Determine the excitatory couplings (arrows) and those couplings that are the same.

For example, the network model for the diagonal experiment in Fig. $4 B$ has four identical attribute columns (representing each of the four stimulus locations) and six types of excitatory arrows (defined by features). More precisely, solid, longdashed, and short-dashed arrows indicate whether the connection is between nodes that are horizontally, vertically, or diagonally aligned. Red and black arrows indicate whether the connection is between nodes stimulated by the same or different colors.

Table 2. Stimulus configurations

\begin{tabular}{lcccc}
\hline \hline & 1 & 2 & 3 & 4 \\
\hline$\alpha$ & RRRR & RGRG & RGGR & RRGG \\
$\beta$ & RGGR & RRGG & RRRR & RGRG \\
$\gamma$ & RRGG & RGGR & RGRG & RRRR \\
$\delta$ & RGRG & RRRR & RRGG & RGGR \\
\hline
\end{tabular}


If no distinction is made between arrows having these two features (orientation of coupling and color of square), then the Wilson network would have more symmetry (rotation by $90^{\circ}$, for example). It would then follow from equivariant Hopf bifurcation that more spatiotemporal patterns of periodic solutions would exist. In particular, these bifurcations would lead to the existence of partially fused states (see Diekman et al. 2013; Diekman and Golubitsky 2014).

Similarly, in the odd configuration $H$, ignoring these features would lead to specific dominant patterned solutions. First, if we ignore the color feature, then the result would be a model with both $\kappa$ and $\tau$ symmetry. The model predictions would be the same for this configuration as the ones for configurations $C-F$.

Second, if the orientation feature is ignored, then the horizontal and vertical excitatory arrows (with the same color) would be identical and the permutation $\sigma=(37)(48)$ would be a symmetry of the Wilson network in Fig. $6 B$. We would then expect two special percepts. In the first, the color in the lower left would be the same as the upper right and the color of the other diagonal locations would be arbitrary. Second, the color in the lower left would be opposite to that in the upper right and the other diagonal locations would be fusion states.

We remark that we could assume that excitatory arrows exist between nodes in different columns that represent the same color (red or green). This possibility was discussed in Diekman and Golubitsky (2014) under the notion of level feature. However, in the rivalry networks we analyze here, the addition of level excitatory arrows will not change the symmetry of the rivalry networks and hence not change the menu of predicted perceptual states. The addition of these couplings might be relevant if simulation of specific Wilson-Cowan equations were contemplated.

\section{MATERIALS AND METHODS}

\section{Observers}

All three observers had normal or corrected-to-normal vision and provided written, informed consent under an institutional review board protocol of The Ohio State University.

\section{Apparatus}

The experiments were conducted on a Dell (Optiplex 980) PC computer using MATLAB R2011b with Psychtoolbox 3.0.10 extensions. The stimuli were presented on the center of a Dell CRT monitor (model no: M993s) and viewed at a distance of $107 \mathrm{~cm}$. Images were presented to the observer's left and right eyes via a stereoscope (Stereo Aids, RdNo70.485) mounted on a chin rest. The monitor was the only light source in the dark room.

\section{Stimuli}

Each colored square extended $112 \times 112$ pixels $\left(1.36^{\circ} \times 1.36^{\circ}\right.$ visual angle at a viewing distance of $107 \mathrm{~cm}$ ). Two colors, red and green, with equal luminance, were used.

Four colored squares centered at the four corners of an imaginary square were presented to each eye via a stereoscope. Each side of the imaginary square extended $2.71^{\circ}$ and $3.17^{\circ}$ in the no-spacing and spacing conditions, respectively. There were two configurations (Fig. 7, $A$ and $B$ ) in the no-spacing experiments and six (Fig. 7, $C-H$ ) in the spacing experiments. Black lines (Fig. 7) were presented around colored squares to help binocular fusion. The black lines were presented throughout each 5-min block.

\section{Design}

Each observer participated in at least one training session before the three main sessions. Each session consisted of eight 5-min blocks, one for each of the eight 2-s presentation experiments (Fig. 7). In each 5-min block, observers reported the simultaneously perceived colors of the four squares that had been presented for $2 \mathrm{~s}$ at the time of each auditory beep in each trial. The 2-s presentation was based on pilot experiments in which rivalry was generally observed before the end of the 2 -s period. In response to the request of an anonymous reviewer, we conducted an additional set of experiments with 4-s presentations on the same three subjects with configurations $C, E, F$, and $G$ (Fig. 7). Each of the same three observers completed two experimental sessions. Each session consisted of eight 5-min blocks, two for each of the four 4-s presentation experiments.

\section{Procedure}

Before each block, observers used four arrow keys (left, right, up, and down) on the computer keyboard to adjust the relative positions of the images (the dark lines) presented to the two eyes until stable binocular fusion was achieved. They then pressed the space bar to initiate the 5-min block. In each trial, the stimulus was presented for $2 \mathrm{~s}$ (or $4 \mathrm{~s}$ in the 4-s presentation experiments), and, upon its termination, an auditory beep cued observers to report the simultaneously perceived colors of the squares at all locations at the time of the auditory cue using the computer keyboard. Counterclockwise, starting from the upper left, observers pressed the left and right arrow keys to report the perceived red or green color. $\mathrm{R}$ or $\mathrm{G}$ appeared at the corresponding location as the observer pressed left or right arrow keys. Observers could delete the letter (R or $G$ ) at the most recently reported location with the Delete key and rereport the response. By pressing the Delete key multiple times, observers could change responses at multiple locations. A new trial started $0.5 \mathrm{~s}$ after the press of the space bar to confirm the responses or after the press of the ESC key to abort the current trial (e.g., due to attention lapse). No feedback was given. Observers were instructed to fixate at the center of the image.

\section{Analysis}

We conducted Student's $t$ test (Wilcox 2012) with MATLAB command ttest on the reported percentage of even percepts in the 12 experiments. Specially, we conducted one-sided Student's $t$ test in experiments with configurations $A-F$ (null hypothesis: $=0.5$; alternative hypothesis: $>0.5$ ) and two-sided Student's $t$ test in experiments with configurations $G$ and $H$ (null hypothesis: $=0.5$ ).

\section{EXPERIMENTAL RESULTS}

In the 2-s presentation experiments, Tables 3, 4, and 5 show the total number of reports of all 16 possible percepts for observers 1, 2, and 3, respectively. The report frequency of different percepts differed between experiments and observers. Two pure color (RRRR, GGGG) percepts and two percepts consistent with the monocular images presented to either the left or right eyes were most often reported. In the no-spacing pure color experiment (Fig. 7A), only two pure color percepts were reported by observers 2 and 3. However, observer 1 also reported vertical (GGRR), horizontal (GRRG), and lower right (GGRG) percepts, suggesting that the black lines might effectively act as "spacing" for this observer. In the no-spacing vertical experiments (Fig. 7B), two pure color (RRRR, GGGG) and two vertical (RRGG, GGRR) percepts contributed to the majority of the reports while the other 12 percepts were rarely 
Table 3. Report frequencies of the 16 possible percepts for observer 1 in all eight 2-s presentation experiments

\begin{tabular}{lrrrrrrrr}
\hline \hline Configuration & $A$ & $B$ & $C$ & $D$ & $E$ & $F$ & $G$ & $H$ \\
\hline RRRR & $59^{*}$ & 33 & $33^{*}$ & 59 & 15 & 20 & 42 & 21 \\
RGRR & 0 & 0 & 15 & 9 & 12 & 19 & 33 & 4 \\
RRGR & 0 & 0 & 2 & 4 & 4 & 5 & 1 & $15^{*}$ \\
RGGR & 1 & 0 & 1 & 1 & $24^{*}$ & 12 & 3 & 7 \\
RRRG & 1 & 2 & 9 & 4 & 10 & 12 & 6 & 14 \\
RGRG & 0 & 0 & 5 & 1 & 12 & $20^{*}$ & 20 & 5 \\
RRGG & 0 & $16^{*}$ & 10 & $10^{*}$ & 0 & 2 & 2 & 1 \\
RGGG & 0 & 0 & 4 & 1 & 5 & 1 & $16 \dagger$ & 1 \\
GRRR & 0 & 15 & 18 & 50 & 22 & 40 & $27 *$ & 22 \\
GGRR & 13 & $138 \dagger$ & 32 & $68 \dagger$ & 10 & 14 & 7 & 20 \\
GRGR & 0 & 1 & 0 & 6 & 16 & $31 \dagger$ & 2 & 11 \\
GGGR & 0 & 1 & 4 & 4 & 9 & 11 & 1 & 12 \\
GRRG & 58 & 1 & 47 & 21 & $104 \dagger$ & 60 & 51 & $62 \dagger$ \\
GGRG & 16 & 1 & 55 & 6 & 10 & 11 & 16 & 60 \\
GRGG & 1 & 10 & 12 & 12 & 8 & 3 & 20 & 5 \\
GGGG & $122 \dagger$ & 37 & $19 \dagger$ & 11 & 3 & 1 & 17 & 2 \\
\hline
\end{tabular}

$\mathrm{G}$ and $\mathrm{R}$ refer to green and red, respectively. The order of the reports was counterclockwise from the top left. Configurations correspond to configurations in Fig. 7. $*$ and $\dagger$ refer to the percepts that are the same as the monocular images presented to the left and right eyes, respectively.

reported. In the six experiments (Fig. 7, $\mathrm{C}-\mathrm{H}$ ) with spacing, report frequency spread over more percepts.

Table 6 shows the individual and mean report percentage of the eight even percepts (pure color, diagonal, horizontal, and vertical) and the $P$ values. In the no-spacing experiments, more than $90 \%$ of the reports were even percepts. In the experiments with spacing, even percepts were reported more often (mean $60.1-72.9 \%$ ) in the even configurations (Fig. 7, $C-F$ ) but around 50\% in the odd configurations (Fig. 7, $G$ and $H$ ). $P$ values are $0.001,0.003,0.068,0.027,0.036,0.006,0.576$, and 0.300 for configurations $A-H$, respectively. This suggests that 1) even percepts were reported significantly more often in all even configurations but configuration $C$ in which the effects were marginally significant; 2 ) even percepts were not significantly more likely to be reported than odd percepts in the odd

Table 4. Report frequencies of the 16 possible percepts for observer 2 in all eight 2 -s presentation experiments

\begin{tabular}{lcrrrrrrr}
\hline \hline Configuration & $A$ & $B$ & $C$ & $D$ & $E$ & $F$ & $G$ & $H$ \\
\hline RRRR & $135^{*}$ & 33 & $110^{*}$ & 66 & 44 & 58 & 47 & 53 \\
RGRR & 0 & 0 & 3 & 9 & 4 & 11 & 5 & 2 \\
RRGR & 0 & 0 & 2 & 14 & 10 & 3 & 2 & $28^{*}$ \\
RGGR & 0 & 2 & 7 & 7 & $48^{*}$ & 6 & 2 & 19 \\
RRRG & 0 & 0 & 7 & 4 & 9 & 6 & 13 & 4 \\
RGRG & 0 & 0 & 2 & 7 & 7 & $12^{*}$ & 5 & 0 \\
RRGG & 1 & $10^{*}$ & 2 & $17^{*}$ & 3 & 3 & 11 & 2 \\
RGGG & 0 & 1 & 0 & 13 & 8 & 2 & $76 \dagger$ & 2 \\
GRRR & 0 & 0 & 7 & 13 & 3 & 20 & $21^{*}$ & 0 \\
GGRR & 0 & $121 \dagger$ & 4 & $23 \dagger$ & 0 & 2 & 0 & 1 \\
GRGR & 0 & 0 & 1 & 14 & 4 & $34 \dagger$ & 3 & 7 \\
GGGR & 0 & 7 & 1 & 12 & 5 & 16 & 2 & 8 \\
GRRG & 0 & 1 & 30 & 3 & $54 \dagger$ & 11 & 21 & 9 \\
GGRG & 0 & 1 & 5 & 9 & 8 & 8 & 2 & $55 \dagger$ \\
GRGG & 1 & 0 & 6 & 12 & 8 & 24 & 10 & 6 \\
GGGG & $122 \dagger$ & 64 & $60 \dagger$ & 17 & 27 & 17 & 22 & 46 \\
\hline
\end{tabular}

$\mathrm{G}$ and $\mathrm{R}$ refer to green and red, respectively. The order of the reports was counterclockwise from the top left. Configurations correspond to configurations in Fig. $7 . *$ and $\dagger$ refer to the percepts that are the same as the monocular images presented to the left and right eyes, respectively.
Table 5. Report frequencies of the 16 possible percepts for observer 3 in all eight 2-s presentation experiments

\begin{tabular}{lcrrrrrrr}
\hline \hline Configuration & $A$ & $B$ & $C$ & $D$ & $E$ & $F$ & $G$ & $H$ \\
\hline RRRR & $108^{*}$ & 61 & $48^{*}$ & 21 & 26 & 10 & 10 & 39 \\
RGRR & 0 & 3 & 12 & 10 & 7 & 10 & 19 & 9 \\
RRGR & 0 & 2 & 3 & 0 & 6 & 6 & 1 & $26^{*}$ \\
RGGR & 1 & 0 & 9 & 0 & $46^{*}$ & 6 & 24 & 14 \\
RRRG & 0 & 2 & 1 & 8 & 1 & 6 & 1 & 2 \\
RGRG & 0 & 4 & 2 & 6 & 5 & $23^{*}$ & 4 & 1 \\
RRGG & 1 & $27^{*}$ & 1 & $48^{*}$ & 5 & 4 & 3 & 4 \\
RGGG & 0 & 0 & 1 & 11 & 6 & 18 & $26 \dagger$ & 2 \\
GRRR & 0 & 10 & 2 & 0 & 4 & 8 & $35^{*}$ & 2 \\
GGRR & 1 & $59 \dagger$ & 50 & $9 \dagger$ & 4 & 1 & 32 & 8 \\
GRGR & 0 & 4 & 3 & 0 & 8 & $3 \dagger$ & 16 & 10 \\
GGGR & 1 & 7 & 29 & 3 & 37 & 2 & 4 & 39 \\
GRRG & 0 & 0 & 0 & 3 & $10 \dagger$ & 39 & 13 & 1 \\
GGRG & 0 & 3 & 7 & 1 & 3 & 27 & 1 & $24 \dagger$ \\
GRGG & 1 & 3 & 1 & 16 & 28 & 21 & 20 & 5 \\
GGGG & $134 \dagger$ & 28 & $65 \dagger$ & 105 & 29 & 49 & 33 & 46 \\
\hline
\end{tabular}

$\mathrm{G}$ and $\mathrm{R}$ refer to green and red, respectively. The order of the reports was counterclockwise from the top left. Configurations correspond to configurations in Fig. 7. * and $\dagger$ refer to the percepts that are the same as the monocular images presented to the left and right eyes, respectively.

configurations. We found similar results in the 4-s presentation experiments (Table 7).

In summary, in the experiments using stimulus configurations $C-F$, even percepts (percepts with an even number of red squares) were more frequently observed than not, whereas in the experiments using stimulus configurations $G$ and $H$, even percepts and odd percepts (percepts with an odd number of red squares) were observed with equal probability. The Wilson network models in which all six types of connections are different was in better agreement with the empirical results than the other models in which some of the six connections are the same. We conclude that coupling between neural representations of input stimulus depends on both the feature values and spatial configuration of the representations. More specifically, we conclude that the excitatory coupling strengths in the horizontal and vertical configurations are different, and the coupling strengths between the same color and between different colors are different.

\section{DISCUSSION}

As in Wilson (2007), Diekman et al. (2013), and Diekman and Golubitsky (2014), we assume that the first step in modeling a rivalry experiment is choosing a rivalry network. In this study, we constructed and analyzed the symmetries of generalized rivalry network models for a number of four-location binocular rivalry stimulus configurations. The development of a novel reporting procedure allowed us to obtained frequencies of all potential perceptual states in eight experiments. We have shown that rivalry networks in which coupling depends on stimulus features and spatial configuration of the nodes can lead (via fusion-breaking Hopf bifurcations) to the prediction of the set of percepts that can be perceived in the experiments.

When considering small-amplitude periodic solutions obtained by Hopf bifurcation there is a mathematical reason for considering only those periodic solutions that bifurcate from a fusion state. Specifically, periodic states obtained by Hopf bifurcation can exhibit alternation only if that bifurcation is from a fusion state. If not, the activity of one oscillating node 
Table 6. Report percentage (\%) of even percepts in all eight 2-s presentation experiments

\begin{tabular}{|c|c|c|c|c|c|c|c|c|}
\hline Configuration & $A$ & $B$ & C & $D$ & E & $F$ & $G$ & $H$ \\
\hline Observer1 & 93.4 & 88.6 & 55.3 & 66.3 & 69.7 & 61.1 & 54.5 & 49.2 \\
\hline Observer2 & 99.6 & 96.3 & 87.4 & 64.2 & 77.3 & 61.4 & 45.9 & 56.6 \\
\hline Mean & 97.4 & 90.3 & 72.9 & 70.0 & 68.7 & 60.1 & 52.1 & 53.0 \\
\hline$P$ & $0.001 *$ & $0.003 *$ & 0.068 & $0.027 *$ & $0.036^{*}$ & $0.006^{*}$ & 0.576 & 0.300 \\
\hline
\end{tabular}

Configurations correspond to configurations in Fig. 7. *Significance level $\alpha=0.05$.

will be larger than the activity of all other oscillating nodes for all time and alternation will not occur. It follows that alternation obtained from small-amplitude periodic solutions can only occur robustly in a model network where fusion states are structurally stable in that network. For this reason, to describe alternation, we require a rivalry network structure that leads to fusion states.

The rivalry experiments we discussed exhibit interocular grouping, that is, components of the left eye and right eye images are combined to achieve a single coherent percept (Lee and Blake 2004; Papathomas et al. 2005). Such groupings occur when learned images can be naturally subdivided and reassembled in other forms. The subdivision process seems to be captured by the attributes and their types (which define node types) and the features (which define excitatory coupling types), as has been shown for the experiments described here. Together the attributes and features define the rivalry networks and their symmetries. The mathematics of symmetry-breaking Hopf bifurcation leads to an enumeration of the likely ways that the subdivisions can be reassembled, that is, to a set of interocular groupings associated with percepts.

In this study, we constructed and tested the predictions of the generalized rivalry network models for six four-location binocular rivalry configurations. Our goal for this paper is to demonstrate that the mathematical approach, namely, symmetry analysis of the generalized rivalry networks, can lead to predictions of perceptual states that should be observed more frequently in psychophysical experiments. The approach can be extended to make predictions for 1) more complicated binocular rivalry configurations that involve more stimulus features and more stimulus locations, and more interesting learned patterns (Hong and Shevell 2006; Kovács et al. 1996), and 2) other bistable or multistable perceptual illusions such as the Necker cube illusion (Necker 1832; Stewart and Golubitsky 2019).

In the current mathematical approach, we have focused on "the menu of possible percepts" predicted by the symmetries of generalized rivalry networks. Moreover, generalized rivalry networks are specified by listing attributes of learned patterns

Table 7. Report percentage (\%) of even percepts in all four 4-s presentation experiments

\begin{tabular}{lcccc}
\hline \hline Configuration & $C$ & $E$ & $F$ & $G$ \\
\hline Observer1 & 75.8 & 73.8 & 67.7 & 60.6 \\
Observer2 & 88.4 & 75.9 & 60.0 & 46.8 \\
Observer3 & 74.1 & 55.9 & 55.9 & 52.0 \\
Mean & 79.4 & 68.5 & 61.2 & 53.1 \\
$P$ & $0.011^{*}$ & $0.050^{*} \dagger$ & $0.042^{*}$ & 0.517 \\
\hline
\end{tabular}

Configurations correspond to configurations in Fig. 7. *Significance level $\alpha=0.05 ; \dagger 0.049861$ is rounded to 0.050 . and by determining those excitatory couplings that are assumed to be the same and those that are assumed to be different. Our percept predictions are based on these modeling assumptions. Note that the symmetry analysis that follows can also make predictions concerning perceptual alternations; these alternations consist of states along the periodic solutions.

Our experiments confirmed that percepts consistent with symmetry-breaking Hopf bifurcation did appear more frequently than other percepts. However, the observed types of alternation between states did not seem to be in agreement with the symmetry predicted alternation. In the future, a systematic experimental approach may obtain more detailed temporal dynamics of binocular rivalry and directly test predictions of the properties of the periodic solutions.

\section{APPENDIX: EQUIVARIANT HOPF BIFURCATION}

A system of differential equations $\dot{X}=F(X)$ has a linearly stable equilibrium at $X_{0}$ if $F\left(X_{0}\right)=0$ and all eigenvalues of the Jacobian matrix $J=(D F)_{X_{0}}$ have negative real parts. If the system depends on a parameter $\mu$, Hopf bifurcation occurs at $\mu=\mu_{0}$ when a complex conjugate pair of simple eigenvalues of $J$ at $\mu_{0}$ has zero real part. Generically, as $\mu$ varies, this complex conjugate pair crosses into the right hand side of the complex plane with nonzero speed. In this case, the equilibrium loses stability and a unique periodic solution with small-amplitude grows around the equilibrium. Such a bifurcation is called supercritical if the periodic solution is stable and subcritical if the periodic solution is unstable.

\section{$\Gamma$-Equivariant}

A matrix $\sigma$ acting on $X \in \mathbb{R}^{n}$ is a symmetry of the vector field $\dot{X}=F(X)$ if $\sigma$ maps solutions to solutions. Being a symmetry is equivalent to the equivariance condition

$$
F(\sigma X)=\sigma F(X) .
$$

Let $\Gamma$ be a group of matrices acting on $X \in \mathbb{R}^{n}$. The vector field $F$ is $\Gamma$-equivariant if Eq. 6 is valid for all $\sigma \in \Gamma$.

\section{Hopf Bifurcation with Symmetry Group $\Gamma$}

Assume $\Gamma$ is abelian, which is the case with all symmetry groups analyzed in this paper, and assume the system $\dot{X}=F(X)$ is $\Gamma$-equivariant. Suppose also that the equilibrium is $\Gamma$-symmetric; that is, $\sigma X_{0}=X_{0}$ for all $\sigma \in \Gamma$. If $X(t)$ is the $T$-periodic solution emanating from this equilibrium, then $\Gamma$ preserves the bifurcating trajectory and $\sigma\{X(t)\}=\{X(t)\}$. It follows that $\sigma\{X(t)\}=X\left(t+\theta_{\sigma} T\right)$ for $\theta_{\sigma} \in S^{1}=[0,1)$; that is, as far as the periodic solution is concerned the action of every symmetry $\sigma$ is compensated for by a phase shift $\theta_{\sigma}$. The set

$$
\left\{\left(\sigma, \theta_{\sigma}\right) \in \Gamma \times S^{1}: \sigma X(t)=X\left(t+\theta_{\sigma} T\right) \text { for all } t \in \mathbb{R}\right.
$$

describes spatiotemporal symmetries of the periodic solution. 
Systems of $\Gamma$-equivariant differential equations have a type of Hopf bifurcation for each irreducible representation of $\Gamma$. If $\sigma=\Gamma$ has order two, which is the case for all nontrivial symmetries in this paper, then there are two possible types of Hopf bifurcation leading to $T$-periodic solutions of the form

$$
\sigma X(t)=X(t) \text { and } \sigma X(t)=X\left(t+\frac{T}{2}\right)
$$

Each rivalry network in this paper has a symmetry $\rho$ that permutes the red and green nodes in each attribute column simultaneously. It follows from $\rho X(t)=X(t)$ that the nodes in each attribute column are equal and the associated periodic solution is a fusion state. So, we may assume that $\rho X(t)=X\left(t+\frac{1}{2} T\right)$ and consequently all percepts have the form that alternation occurs between complementary red and green patterns.

If $\Gamma=\mathbf{Z}_{2}(\rho) \oplus \mathbf{Z}_{2}(\tau)$, then there are two types of Hopf bifurcation leading to nonfusion percepts and they have the form

$$
\text { (a) } \tau X(t)=X(t) \text { or }(b) \tau X(t)=X\left(t+\frac{T}{2}\right)
$$

If $\Gamma=\mathbf{Z}_{2}(\rho) \oplus \mathbf{Z}_{2}(\tau) \oplus \mathbf{Z}_{2}(\kappa)$, then there are four types of Hopf bifurcation leading to eight nonfusion percepts and they have the form

$$
\begin{array}{ccc}
\text { (a) } & \kappa X(t)=X(t) & \tau X(t)=X(t) \\
\text { (b) } & \kappa X(t)=X(t) & \tau X(t)=X\left(t+\frac{T}{2}\right) \\
\text { (c) } & \kappa X(t)=X\left(t+\frac{T}{2}\right) & \tau X(t)=X(t) \\
\text { (d) } & \kappa X(t)=X\left(t+\frac{T}{2}\right) & \tau X(t)=X\left(t+\frac{T}{2}\right)
\end{array}
$$

The networks described in this paper have abelian symmetry groups $\mathbf{Z}_{2}(\rho), \mathbf{Z}_{2}(\rho) \oplus \mathbf{Z}_{2}(\tau)$, or $\mathbf{Z}_{2}(\rho) \oplus \mathbf{Z}_{2}(\tau) \oplus \mathbf{Z}_{2}(\kappa)$ and the rivalry percepts are characterized by the spatiotemporal symmetries in Eqs. 7 , 8 , or 9 .

\section{ACKNOWLEDGMENTS}

Present addresses for Z. L. Lu: Division of Arts and Sciences, NYU Shanghai, 1555 Century Avenue, Shanghai, 200122, China; Center for Neural Science and Department of Psychology, New York University, 4 Washington Place, New York, NY 10003.

Present address for Y. Zhao: Center for Neural Science, New York University, 4 Washington Place, New York, NY 10003.

\section{GRANTS}

M. Golubitsky was supported in part by NSF Grant DMS-1440386 to the Mathematical Biosciences Institute. Y. Wang was supported by NSF Grants HRD-1800406 and CNS-1831980. Z. Lu and Y. Zhao were supported by National Eye Institute Grants EY021553 and EY017491.

\section{DISCLOSURES}

No conflicts of interest, financial or otherwise, are declared by the authors.

\section{AUTHOR CONTRIBUTIONS}

M.G., Y.W., and Z.-L.L. conceived and designed research; M.G., Y.Z., Y.W., and Z.-L.L. analyzed data; M.G., Y.Z., Y.W., and Z.-L.L. interpreted results of experiments; M.G., Y.Z., and Z.-L.L. prepared figures; M.G., Y.Z., and Z.-L.L. drafted manuscript; M.G., Y.Z., Y.W., and Z.-L.L. edited and revised manuscript; M.G., Y.Z., Y.W., and Z.-L.L. approved final version of manuscript; Y.Z. performed experiments.

\section{REFERENCES}

Alais D. Binocular rivalry: competition and inhibition in visual perception. Wiley Interdiscip Rev Cogn Sci 3: 87-103, 2012. doi:10.1002/wcs.151.

Alais D, Blake R. Grouping visual features during binocular rivalry. Vision Res 39: 4341-4353, 1999. doi:10.1016/S0042-6989(99)00146-7.

Blake R. A primer on binocular rivalry, including current controversies. Brain Mind 2: 5-38, 2001. doi:10.1023/A:1017925416289.

Blake R, O'Shea RP, Mueller TJ. Spatial zones of binocular rivalry in central and peripheral vision. Vis Neurosci 8: 469-478, 1992. doi:10.1017/ S0952523800004971.

Blake R, Wilson H. Binocular vision. Vision Res 51: 754-770, 2011. doi:10. 1016/j.visres.2010.10.009.

Brascamp JW, Klink PC, Levelt WJM. The "laws" of binocular rivalry: 50 years of Levelt's propositions. Vision Res 109: 20-37, 2015. doi:10.1016/ j.visres.2015.02.019.

Curtu R, Shpiro A, Rubin N, Rinzel J. Mechanisms for frequency control in neuronal competition models. SIAM J Appl Dyn Syst 7: 609-649, 2008. doi:10.1137/070705842.

Dayan P. A hierarchical model of binocular rivalry. Neural Comput 10: 1119-1135, 1998. doi:10.1162/089976698300017377.

Diekman C, Golubitsky M, McMillen T, Wang Y. Reduction and dynamics of a generalized rivalry network with two learned patterns. SIAM J Appl Dyn Syst 11: 1270-1309, 2012. doi:10.1137/110858392.

Diekman CO, Golubitsky M. Network symmetry and binocular rivalry experiments. J Math Neurosci 4: 12, 2014. doi:10.1186/2190-8567-4-12.

Diekman CO, Golubitsky M, Wang Y. Derived patterns in binocular rivalry networks. J Math Neurosci 3: 6, 2013. doi:10.1186/2190-8567-3-6.

Dieter KC, Sy JL, Blake R. Individual differences in sensory eye dominance reflected in the dynamics of binocular rivalry. Vision Res 141: 40-50, 2017. doi:10.1016/j.visres.2016.09.014.

Drenhaus U. Ein Beitrag zur Taxonomie der Lemuriformes Gregory 1915 unter besonderer Berücksichtigung der Gattung Lepilemur I. Geoffroy 1851 (dissertation). Kiel, Germany: Universität Kiel, 1975.

Freeman AW. Multistage model for binocular rivalry. J Neurophysiol 94: 4412-4420, 2005. doi:10.1152/jn.00557.2005.

Golubitsky M, Romano D, Wang Y. Network periodic solutions: patterns of phase-shift synchrony. Nonlinearity 25: 1045-1074, 2012. doi:10.1088/ 0951-7715/25/4/1045.

Golubitsky M, Stewart I. The Symmetry Perspective: From Equilibrium to Chaos in Phase Space and Physical Space. Basel, Switzerland: Birkhauser, 2002.

Golubitsky M, Stewart I. Nonlinear dynamics of networks: the groupoid formalism. Bull Am Math Soc 43: 305-364, 2006. doi:10.1090/S0273-097906-01108-6.

Hayashi R, Maeda T, Shimojo S, Tachi S. An integrative model of binocular vision: a stereo model utilizing interocularly unpaired points produces both depth and binocular rivalry. Vision Res 44: 2367-2380, 2004. doi:10.1016/ j.visres.2004.04.017.

Hohwy J, Roepstorff A, Friston K. Predictive coding explains binocular rivalry: an epistemological review. Cognition 108: 687-701, 2008. doi:10. 1016/j.cognition.2008.05.010.

Hollins M, Hudnell K. Adaptation of the binocular rivalry mechanism. Invest Ophthalmol Vis Sci 19: 1117-1120, 1980.

Hong SW, Shevell SK. Resolution of binocular rivalry: perceptual misbinding of color. Vis Neurosci 23: 561-566, 2006. doi:10.1017/S0952523806233145.

Jacot-Guillarmod A, Wang Y, Pedroza C, Ogmen H, Kilpatrick Z, Josić K. Extending Levelt's Propositions to perceptual multistability involving interocular grouping. Vision Res 133: 37-46, 2017. doi:10.1016/j.visres. 2016.12.017.

Kakimoto Y, Aihara K. Hierarchical spatio-temporal dynamics of a chaotic neural network for multistable binocular rivalry. New Math Nat Comput 05: 123-134, 2009. doi:10.1142/S1793005709001301.

Kang MS, Blake R. An integrated framework of spatiotemporal dynamics of binocular rivalry. Front Hum Neurosci 5: 88, 2011. doi:10.3389/fnhum. 2011.00088.

Klink PC, Brascamp JW, Blake R, van Wezel RJA. Experience-driven plasticity in binocular vision. Curr Biol 20: 1464-1469, 2010. doi:10.1016/ j.cub.2010.06.057.

Klink PC, van Ee R, Nijs MM, Brouwer GJ, Noest AJ, van Wezel RJA. Early interactions between neuronal adaptation and voluntary control determine perceptual choices in bistable vision. J Vis 8: 16.1-16.18, 2008. doi:10.1167/8.5.16. 
Kovács I, Papathomas TV, Yang M, Fehér A. When the brain changes its mind: interocular grouping during binocular rivalry. Proc Natl Acad Sci USA 93: 15508-15511, 1996. doi:10.1073/pnas.93.26.15508.

Laing CR, Chow CC. A spiking neuron model for binocular rivalry. J Comput Neurosci 12: 39-53, 2002. doi:10.1023/A:1014942129705.

Lee SH, Blake R. A fresh look at interocular grouping during binocular rivalry. Vision Res 44: 983-991, 2004. doi:10.1016/j.visres.2003.12.007.

Logothetis NK, Leopold DA, Sheinberg DL. What is rivalling during binocular rivalry? Nature 380: 621-624, 1996. doi:10.1038/380621a0.

Meenes M. A phenomenological description of retinal rivalry. Am J Psychol 42: 260-269, 1930. doi:10.2307/1415275.

Necker LA. Observations on some remarkable optical phenomena seen in Switzerland; and on an optical phenomenon which occurs on viewing a figure of a crystal or geometrical solid. Lond Edinb Philos Mag J Sci 1: 329-337, 1832. doi:10.1080/14786443208647909.

O'Shea RP, Blake R, Wolfe JM. Binocular rivalry and fusion under scotopic luminances. Perception 23: 771-784, 1994. doi:10.1068/p230771.

O'Shea RP, Sims AJ, Govan DG. The effect of spatial frequency and field size on the spread of exclusive visibility in binocular rivalry. Vision Res 37: 175-183, 1997. doi:10.1016/S0042-6989(96)00113-7.

Papathomas TV, Kovacs I, Conway T. Interocular grouping in binocular rivalry: basic attributes and combinations. In: Binocular Rivalry, edited by Alais D, Blake R. Cambridge, MA: MIT Press, 2005, p. 155-168.

Richard B, Chadnova E, Baker DH. Binocular vision adaptively suppresses delayed monocular signals. Neuroimage 172: 753-765, 2018. doi:10.1016/ j.neuroimage.2018.02.021.

Said CP, Heeger DJ. A model of binocular rivalry and cross-orientation suppression. PLOS Comput Biol 9: e1002991, 2013. doi:10.1371/journal.pcbi.1002991.

Seely J, Chow CC. Role of mutual inhibition in binocular rivalry. J Neurophysiol 106: 2136-2150, 2011. doi:10.1152/jn.00228.2011.

Shpiro A, Curtu R, Rinzel J, Rubin N. Dynamical characteristics common to neuronal competition models. J Neurophysiol 97: 462-473, 2007. doi:10. 1152/jn.00604.2006.
Stewart I, Golubitsky M. Symmetric networks with geometric constraints as models of visual illusions. Symmetry (Basel) 11: 799, 2019. doi:10.3390/ sym 11060799

Stewart I, Parker M. Periodic dynamics of coupled cell networks I: rigid patterns of synchrony and phase relations. Dyn Syst 22: 389-450, 2007. doi:10.1080/14689360701450410.

Stollenwerk L, Bode M. Lateral neural model of binocular rivalry. Neural Comput 15: 2863-2882, 2003. doi:10.1162/089976603322518777.

Tong F, Meng M, Blake R. Neural bases of binocular rivalry. Trends Cogn Sci 10: 502-511, 2006. doi:10.1016/j.tics.2006.09.003.

van Ee R. Stochastic variations in sensory awareness are driven by noisy neuronal adaptation: evidence from serial correlations in perceptual bistability. J Opt Soc Am A Opt Image Sci Vis 26: 2612-2622, 2009. doi:10. 1364/JOSAA.26.002612.

Wade NJ. Early studies of eye dominances. Laterality 3: 97-108, 1998. doi:10.1080/713754296.

Whittle P, Bloor D, Pocock S. Some experiments on figural effects in binocular rivalry. Percept Psychophys 4: 183-188, 1968. doi:10.3758/ BF03210465.

Wilcox R. Hypothesis testing. In: Modern Statistics for the Social and Behavioral Sciences: A Practical Introduction. Boca Raton, FL: CPC, 2012, p. 193-195.

Wilson HR. Minimal physiological conditions for binocular rivalry and rivalry memory. Vision Res 47: 2741-2750, 2007. doi:10.1016/j.visres.2007.07. 007.

Wilson HR. Requirements for conscious visual processing. In: Cortical Mechanisms of Vision, edited by Jenkin MRM, Harris LR. New York: Cambridge University Press, 2009, p. 217-235.

Wilson HR. Binocular rivalry: neurons unwire when they can't simultaneously fire. Curr Biol 20: R715-R717, 2010. doi:10.1016/j.cub.2010.07.015.

Wilson HR. Binocular contrast, stereopsis, and rivalry: toward a dynamical synthesis. Vision Res 140: 89-95, 2017. doi:10.1016/j.visres.2017.07. 016. 